\title{
A necessary and sufficient condition for convergence to equilibrium in Kolmogorov systems
}

Esteban Calzetta

Citation: Journal of Mathematical Physics 32, 2903 (1991); doi: 10.1063/1.529083

View online: https://doi.org/10.1063/1.529083

View Table of Contents: http://aip.scitation.org/toc/jmp/32/10

Published by the American Institute of Physics

\section{PHYSICS TODAY}

MANACER'S GUIDE

WHITEPAPERS
Accelerate R\&D with Multiphysics Simulation
READ NOW

PRESENTED BY フ๐ С๐MSOL 


\title{
A necessary and sufficient condition for convergence to equilibrium in Kolmogorov systems
}

\author{
Esteban Calzetta \\ Instituto de Astronomía y Fisica del Espacio, Casilla de Correo 67-Sucursal 28, 1428 Buenos Aires, \\ Argentina and Departamento de Fisica, Facultad de Ciencias Exactas y Naturales, Universidad de Buenos \\ Aires, Ciudad Universitaria, Pabellón I, 1428 Buenos Aires, Argentina
}

(Received 29 October 1990; accepted for publication 14 May 1991)

\begin{abstract}
A necessary and sufficient condition is established for a state to converge to equilibrium in a dynamical system described as a Kolmogorov system. Because the time reversal of a state converging to equilibrium does not necessarily share this property, this opens the possibility of spontaneous breaking of time reversal symmetry.
\end{abstract}

\section{INTRODUCTION}

According to the conventional view, the physical Universe is composed of two tiers, a microscopic level described by deterministic, time reversal invariant laws, and a macroscopic level described in probabilistic, time directed terms. The former is the sphere of Classical Mechanics and its heirs, such as Quantum Mechanics and Quantum Field Theory, while the latter is the preserve of Equilibrium and Nonequilibrium Statistical Mechanics. The borderline between the two levels was considered observer dependent, in the sense that it was the ignorance (on the part of the observer) of the true microscopic state of a physical system which caused the breakdown of the deterministic description, and its necessary replacement by the nondeterministic point of viewhere, of course, we regard Quantum Mechanics as deterministic, in the sense that the time evolution of the state of an isolated system is both deterministic and time reversal invariant.

It is by now well established that this view of Nature is oversimplified. There is a well-known set of dynamical systems whose trajectories, while being deterministic, are so complex that they cannot be distinguished in any significant way from those resulting from stochastic processes. ${ }^{1}$ In some of these systems we even observe two phenomena long thought incompatible with deterministic, time reversal invariant dynamics: convergence to equilibrium and spontaneous time symmetry breaking. ${ }^{2}$ By this last phenomenon we mean the existence of states converging to equilibrium only when time flows in some well-defined sense. The ensuing breaking of time reversal invariance-the two directions of time can now be physically distinguished-is spontaneous, in the same sense that we apply this word to the breaking of rotation invariance in a ferromagnet.

The implications of the existence of spontaneous time symmetry breaking are far reaching. If it were possible to show that the Universe itself, considered as a dynamical system, is "chaotic" enough, it could even lead to a new understanding of the second law of thermodynamics, in which this law is deduced from time symmetry breaking occurring on a cosmic scale. ${ }^{3}$ It is therefore interesting to point out as clearly as possible which dynamical systems and what classes of states may manifest behavior of this kind.

In this paper we shall study time symmetry breaking in the class of Kolmogorov systems. ${ }^{4}$ The existence of time asymmetric states in these systems has been shown by Courbage. ${ }^{5}$ We shall continue his investigation by stating a condition which is both necessary and sufficient for a state to converge to equilibrium (in a sense to be specified below). The class of states satisfying this condition is not closed under time reversal, in agreement with earlier results.

The remainder of this paper is organized as follows. We have included in the next section some of the necessary background on Dynamical Systems theory. In Sec. III we state and prove our main results. We also compare our proposed condition for convergence to equilibrium with earlier work by Courbage. ${ }^{5}$ We conclude in Sec. IV with some brief remarks on the physical relevance of our results.

\section{DYNAMICAL SYSTEMS}

The statement of our main results in the next section requires the introduction of some concepts from ergodic theory. ${ }^{4}$ We have included in this section a brief review of this background material.

Following Arnold and Avez, ${ }^{4}$ we shall call a triad $(M, \mu, \phi)$ an "abstract dynamical system" (ADS), provided $\mu$ is a probability measure on the space $M$ and $\phi$ is a measure preserving automorphism (we shall state the relevant definitions and theorems in the discrete case only; the generalization to the continuous case is more or less immediate). We may define several functional spaces, such as the space $L^{1}$ of summable functions with respect to the measure $\mu$, the space $L^{2}$ of square integrable functions, the space $L^{\infty}$ of functions bounded almost everywhere (a.e.), etc. The automorphism $\phi$ induces a transformation $U$ in these spaces, according to the rule $[U f](x)=f[\phi(x)]$. $U$ preserves the natural norm on each of these spaces, and in particular it is unitary on $L^{2}$.

An "observable" shall be any real, bounded function on $M$, and a "state" a probability measure. We may use two different representations of the dynamics. In the "Heisenberg picture," the state of the system is given once and for all, and observables evolve in (discrete) time according to the transformation $U$. In the "Schrodinger picture" the observables remain constant, but states evolve. Namely, the state $\rho$ evolves into the measure $U^{\dagger} \rho$, defined as $U^{\dagger} \rho(A)=\rho\left(\phi^{-1} A\right)$ for every measurable set $A$. 

and $B$,

An ADS is called "mixing" if for any measurable sets $A$

$\mu\left(\phi^{n} A \cap B\right) \rightarrow \mu(A) \mu(B)$.

When $n \rightarrow \infty$. If a system is mixing, then we find

$\int_{M} d \mu\left(U^{n} f\right) g \rightarrow \int_{M} d \mu f \int_{M} d \mu g$.

For large enough $n$ whenever $f$ and $g$ belong to $L^{z}$ or else if $f \in L^{1}$ and $g \in L^{\infty}$.

Mixing systems are "chaotic" enough to allow us to discuss convergence to equilibrium in a certain sense, within the framework of the deterministic evolution of the ADS. Effectively, consider a state defined by a measure $\rho$ of the form $\rho(A)=\int_{A} \rho(x) d \mu$, where $\rho(x)$ is some $L^{1}$ function (these measures are called "absolutely continuous" with respect to $\mu)$. Then it follows immediately from (2) that $\left(U^{\dagger}\right)^{n} \rho(A) \sim \mu(A)$ for any measurable set $A$ if $n$ is large enough. More physically, this means that after a long enough time, the probability of finding the system within a subset $A$ of $M$ converges to its equilibrium valuc. The same holds for the mean value of any observable. We shall call this way of approaching equilibrium "convergence in the weak$\operatorname{star}\left(W^{*}\right)$ sense."

We must stress that the convergence to equilibrium of certain ("fuzzy" enough) states in this sense is a result of the dynamics itself, even if this dynamics is time reversal invariant, and involves no approximations nor "coarse graining" of the space $M$. On the other hand, the existence of states converging to equilibrium in the $W^{*}$ sense does not guarantee time symmetry breaking. An absolutely continuous state, for example, will converge to equilibrium as discrete time goes to infinity in either sense.

\section{CONDITIONS FOR TIME SYMMETRY BREAKING}

In this section we show the necessary and sufficient condition for a state $\rho$ to converge to equilibrium (condition TSB below), and the relationship of this condition to spontaneous time symmetry breaking. We also compare these results to earlier work by Courbage. ${ }^{5}$

To state our main result we must restrict ourselves to a subclass of mixing systems with stronger unstability properties. These are the so-called Kolmogorov (K) systems. ${ }^{4}$

An ADS is called a $\mathrm{K}$ system if there exists a $\sigma$ algebra $\mathscr{A}$ with the following properties: (i) $\mathscr{A} \subset \phi \mathscr{A}$ (ii) $\cap_{n} \phi^{n} \mathscr{A} \subset \hat{0}$ (iii) The $\sigma$ algebra generated by all the $\phi^{n} \cdot \mathscr{A}$ equals $\hat{1}$.

Here $\hat{0}$ and $\hat{1}$ are the $\sigma$ algebras generated by the measure 0 sets and the measurable sets, respectively.

With respect to this definition, we observe that Arnold and $A v e z^{4}$ actually require equality in (ii); we adopt this more relaxed definition as we think it simplifies the proofs below with no significant loss of generality.

Any $\mathrm{K}$ system is mixing, ${ }^{4}$ and so there are states that converge to equilibrium. Courbage ${ }^{5}$ has shown that, if the restriction $\rho_{M}$ of $\rho$ to $\mathscr{A}_{M}=\phi^{-M} . \mathscr{A}$ is absolutely continuous for some $M$, then the state $\rho$ converges to the equilibrium distribution $\mu$ in the $W^{*}$ sense.

Moreover, if a state $\rho$ satisfies Courbage's criterion for convergence, then there is a summable function $\rho_{M}(x)$ associated with any of its absolutely continuous restrictions. Then, after choosing some $M$, we can associate to $\rho$ the nonequilibrium entropy

$$
S[\rho]=\int d \mu \rho_{M}(x) \ln \rho_{M}(x) .
$$

Courbage 5 has shown that $S$ is nondecreasing. However, we must point out that there may be absolutely continuous states with infinite entropy which nevertheless converge to equilibrium.

Courbage's criterion for convergence may be stated formally as "a state $\rho$ will converge to $\mu$ in the $W$ * sense if there is an $N$ such that, for any set $A \in \mathscr{A}_{N}, \mu(A)=0$ implies $\rho(A)=0$." This condition, however, is not necessary. We now proceed to state a necessary and sufficient condition for convergence. This is: (TSB) "A state $\rho$ will converge to $\mu$ in the $W^{*}$ sense if and only if for every $\epsilon>0$ there is an $N$ such that, for any set $A \in \mathscr{A}_{N}, \mu(A)=0$ implies $\rho(A) \leqslant \epsilon$."

To see that condition (TSB) is necessary, suppose that, for some $\epsilon>0$, we could choose, in each $\mathscr{A}_{N}(N \geqslant 0)$, a set $A_{N}$ such that $\mu\left(A_{N}\right)=0$ but $\rho\left(A_{N}\right) \geqslant \epsilon$. Let $A=\cup_{N} \phi^{N} A_{N}$. Then $\mu(A)=0$, but $U^{\dagger N} \rho(A) \geqslant \rho\left(A_{N}\right) \geqslant \epsilon$. Therefore, $\rho$ cannot possibly converge to equilibrium.

To see that condition (TSB) is sufficient we must recall the following facts. We shall call a real, bounded, $\sigma$ additive function on the measurable subsets of $M$ a "charge." Of course, a positive charge is simply a measure. Given a charge $\sigma, M$ can be decomposed into two sets $A$ and $B$ such that, for any set $C, \sigma(C \cap A) \geqslant 0$, and $\sigma(C \cap B) \leqslant 0$. The sets $A$ and $B$ are called positive and negative, respectively. The separation of $M$ into these two sets is called the "IIahn decomposition."

We can show that, for any measure $\rho$, there is a set $B$ such that $\mu(B)=0$, and the measure $\rho_{R}(C)=\rho\left(C \cap B^{c}\right)$ is absolutely continuous [ that is, $\mu(C)=0 \Rightarrow \rho_{R}(C)=0$ ].

In effect, consider the Hahn decomposition of $M$ into sets $A_{N}$ and $B_{N}$, positive and negative for the charges $\rho_{N}=N \mu-\rho, N \geqslant 1$. Let $B=\cap B_{N}$. Then for every $N$ we find $\mu(B) \leqslant(1 / N) \rho(B) \leqslant(1 / N)$, so $\mu(B)=0$. Moreover, for every set $C$ we find

$$
\begin{aligned}
\rho\left(C \cap B^{c}\right) & =\rho\left(\cup\left(C \cap B_{N}^{c}\right)\right) \\
& \leqslant \sum \rho\left(C \cap B_{N}^{c}\right) \leqslant \sum N \mu\left(C \cap B_{N}^{c}\right) .
\end{aligned}
$$

Thus $\mu(C)=0 \Rightarrow p_{R}(C)=0$.

This result can be phrased as: every measure $\rho$ can be written in the form $\rho=\rho_{R}+\rho_{S}$, where $\rho_{R}$ is absolutely continuous and $\rho_{S}(C)=\rho(C \cap B)$, where $\mu(B)=0$.

If moreover $\rho$ satisfies condition (TSB) above, we obtain that for every $\epsilon$ there is an $N$ such that $\rho_{S}(C) \leqslant \epsilon$ for any $C \in \mathscr{L}_{N}$.

Now let $\rho$ satisfy condition (TSB), choose $\epsilon>0$ and let $N$ be the corresponding integer. Let $C \in \mathscr{A}_{M}$. For $n \geqslant N-M$, $C_{n}=\phi^{-n} C \in \mathscr{A}_{N}$, and $\rho\left(C_{n}\right)=p_{N}\left(C_{n}\right)=\rho_{N R}\left(C_{n}\right)$ $+\rho_{N S}\left(C_{n}\right) \leqslant \rho_{N R}\left(C_{n}\right)+\epsilon$. On the other hand, since $\rho_{N R}$ is absolutely continuous, $\rho_{N R}\left(C_{n}\right) \rightarrow \rho_{N R}(M) \mu(C)$ when $n \rightarrow \infty$. Now clearly $1 \geqslant \rho_{N R}(M) \geqslant 1-\epsilon$. Therefore, $\rho\left(C_{n}\right) \rightarrow \mu(C)$.

If the set $C$ does not belong to any $\mathscr{A}_{M}$, there is never- 
theless some $N$ and sets $E \subset C \subset F$ in $\mathscr{A}_{N}$ such that $\mu(F-E) \leqslant \epsilon$. Applying the above result to $E$ and $F$ we find that $\rho\left(\phi^{-n}(C)\right) \rightarrow \mu(C)$ as $n \rightarrow \infty$ also in this case.

This concludes the proof of the sufficiency of condition (TSB) for convergence to equilibrium. We stress the timeasymmetric character of this condition, as only the behavior of the measure $\rho$ on the $\sigma$ algebras $\mathscr{A}_{N}$ for large $N$ is involved. Thus it is easy to construct states which satisfy the condition above, while their time reversal does not.

(TSB) actually reduces to Courbage's condition for convergence to equilibrium in the important class of $\mathrm{K}$ systems known as "Bernoulli shifts." 6.7

A Bernoulli shift is an ADS where $M$ is the set of infinite sequences $\left\{k_{j},-\infty \leqslant j \leqslant \infty\right\}$ of elements of the set $Z_{N+1}=\{0,1, \ldots, N\}$.

To define a measure $\mu$ on $M$ we introduce a measure $\left(p_{0}, p_{1}, \ldots, p_{N}\right)$ on $Z_{N+1}$. Then the measure of the "cylinder" set $A_{j}^{i}=\left\{k_{n} / k_{j}=i\right\}$ is defined as $p_{i}$, the measure of a nonempty finite intersection of cylinders (these sets are also called cylinders) as the product of the measures, and the measure of disjoint unions is defined by additivity. The cylinders generate the $\sigma$ algebra $\hat{1}$ of measurable sets. The Bernoulli shift corresponding to this choice of measure is identified as $B\left(p_{0}, \ldots, p_{N}\right)$.

Finally the automorphism $\phi$ is the shift $(\phi k)_{j}=k_{j+1}$.

The $\sigma$ algebra which makes a Bernoulli shift a K-system is the $\sigma$ algebra $\mathscr{A}_{0}$ generated by the cylinders $A{ }_{j}^{i}$ with $j \geqslant 0$. As above, we shall call $\mathscr{A}_{M}=\phi^{-M} \mathscr{A}_{0}$. This is also the $\sigma$ algebra generated by the cylinders with $j \geqslant M{ }^{4}$

If a given set $C \in \mathscr{A}_{N}$ and $\mu(C)=0$, we may define the new set $C_{1}$ of those sequences which differ from the elements of $C$ at most in their $N$ th entry. It is clear that $C_{1} \in \mathscr{A}_{N+1}$, $\mu\left(C_{1}\right)=0$, and $C \subset C_{1}$. Recursively, we may define a whole increasing sequence $C_{n}$ of measure zero sets, such that $C_{n} \in \mathscr{d}_{N+n}$ for each $n$. If the restriction to $\mathscr{A}_{N}$ of a measure $\rho$ is not absolutely continuous, then there is a set $C$ in $\mathscr{A}_{N}$ such that $\mu(C)=0$ and $\rho(C) \neq 0$. But then in each $\mathscr{A}_{N+n}$ we find the corresponding set $C_{n}$ and $\rho\left(C_{n}\right) \geqslant \rho(C)$, so condition (TSB) is not satisfied. This shows the equivalence between this condition and absolute continuity.

We shall conclude this section with a concrete example, the Bernoulli shift $B(1 / 2,1 / 2)$. This is the set of doubly infinite sequences of coin flips, with heads and tails having the same probability. Clearly, all the theory we have developed thus far can be immediately applied to this case.

The $B(1 / 2,1 / 2)$ furthermore has an interesting geometric interpretation. Consider the sequence

$$
\left(\ldots, k_{-1}, k_{0}, k_{1}, \ldots\right)
$$

and form the two real numbers

$$
\begin{aligned}
& x=\sum_{j>0} \frac{k_{j}}{2^{j+1}}, \\
& y=\sum_{j>1} \frac{k_{-j}}{2^{j}} .
\end{aligned}
$$

Equations (5) and (6) define a transformation from $M$ to the unit square, under which the equilibrium measure $\mu$ becomes the Lebesgue measure. The shift $\phi$ becomes the transformation

$$
\phi(x, y)=(2 x(\bmod 1),(y+[2 x]) / 2) .
$$

(Where [ ] denotes the integer part). This transformation is known as the "Baker's transformation."2,8

The cylinders $A_{j}^{i}$ are sets of horizontal stripes if $j\langle 0$; there are $2^{-j-1}$ equally spaced stripes of width $2^{j}$. If $j \geqslant 0$, the stripes are vertical. As $\phi\left(A_{j}^{i}\right)=A_{j-1}^{i}$, the Baker's transform turns "fences" into "ladders." The time reversal operation is the exchange of $x$ and $y$.

Points along a vertical line tend to converge under the evolution; these are the so-called "contracting fibers." On the contrary, points along a horizontal line tend to be scattered all over the square; these are the "dilating fibers." States concentrated on dilating fibers (but smooth in the $x$ direction) converge to equilibrium. States concentrated on contracting fibers do not tend to equilibrium. This, therefore, shows that time symmetry can be spontaneously broken in this dynamical system.

\section{FINAL REMARKS}

In this paper we have stated a necessary and sufficient condition for convergence to equilibrium in a Kolmogorov system. The condition obtained is time asymmetric. Indeed, time symmetry is spontaneously broken by states converging to equilibrium only in a given sense of time.

It seems fair to say that for the time being the relevance of these results to physics is mainly conceptual, in that they highlight an aspect of the relationship between irreversible behavior and the microscopic laws of motion which has been unduly neglected in the past. The states which actually break time symmetry are highly singular with respect to the equilibrium measure of the dynamical system, and therefore it is not clear that they play a major role in ordinary irreversible behavior as it is studied in Thermodynamics. States which are absolutely continuous with respect to the equilibrium measure satisfy condition TSB above, and therefore converge to equilibrium in the weak-star sense, but they do so in either time direction.

In this connection it is worth noting another remarkable characteristic of Kolmogorov systems, that they allow for the definition of a (Kolmogorov) "entropy" depending on the dynamics of the system alone, without invoking either coarse graining or lack of knowledge on the part of the observer. The exact relationship of Kolmogorov entropy to the usual thermodynamical one is a subject of active research. ${ }^{9}$

Moreover, Kolmogorov systems are worth studying on their own right, as many chaotic systems contain invariant subsystems belonging to this class. ${ }^{10}$

To conclude, we would like to suggest an important but somewhat unconventional field in which spontaneous time symmetry breaking may be relevant. This is the origin of irreversible evolution in Cosmology.

As we mentioned in the Introduction, spontaneous time symmetry breaking may have occurred in the early stages of the cosmic evolution. It is well known that according to present observations our Universe appears to be highly homogeneous and isotropic now, but it was not necessarily so earlier in its evolution. If one relaxes the requirement of isotropy, while considering only homogeneous Universes, there are 
strong theoretical arguments ${ }^{11}$ leading to the conclusion that, close enough to the initial singularity or Big Bang, the Universe may be described by a so-called Bianchi type IX model. ${ }^{12}$ These models are known to be chaotic, and indeed they can be closely approximated by a Bernoulli shift. ${ }^{13}$

Through spontaneous time symmetry breaking, chaos in the Bianchi type IX models may be responsible for a new "arrow of time" alongside the already known cosmological, electromagnetic and thermodynamical ones. ${ }^{14}$ Moreover, if a connection between this "chaotic" arrow of time and the other ones could be demonstrated, this would give new content to Penrose's conjecture that the direction of time flow has been determined by the state of the Universe in its earliest stages of development. ${ }^{15}$ We shall delve into this topic in greater detail elsewhere. ${ }^{3}$

\section{ACKNOWLEDGMENTS}

This work has been supported in part by CONICET and Universidad de Buenos Aires, and by the Directorate General for Science, Research and Development of the Commission of the European Communities under contract No. C 110540-M(TT).
'V. M. Alekseev and M. V. Yakobson, Phys. Rep. C 75, 287 (1981).

${ }^{2}$ I. Prigogine, From Being to Becoming (W. H. Freeman, San Francisco, 1980).

${ }^{3}$ E. Calzetta, GTCRG preprint (1990).

${ }^{4}$ V. I. Amold and A. Avez, Ergodic Problems of Classical Mechanics (Benjamin, New York, 1968).

${ }^{5}$ M. Courbage, Physica A 122, 459 (1983).

${ }^{6} \mathrm{P}$. Shields, The Theory of Bernoulli Shifts (University of Chicago, Chicago, IL, 1974).

${ }^{7}$ B. Misra, I. Prigogine, and M. Courbage, Physica A 98, 1 (1979); B. Misra and M. Courbage, Physica A 104, 359 (1980).

${ }^{8}$ I. Prigogine and Y. Elskens, in Quantum Implications, edited by B. J. Hiley and F. David-Peat (Routledge, London, 1987).

${ }^{9}$ P. Gaspard and G. Nicolis, Phys. Rev. Lett. 65, 1693 (1990).

${ }^{10} \mathrm{~J}$. Guckenheimer and P. Holmcs, Nontinear Oscillations, Dynamical Systems, and Bifurcations of Vector Fields (Springer-Verlag, Heidelberg, 1983).

"V. A. Belinskii, E. M. Lifshitz, and Z. M. Khalatnikov, Adv. Phys. 19, 525 (1970); C. W. Misner, in Relativity, edited by M. Carmeli (Plenum, New York, 1970), p. 55.

${ }^{12} \mathrm{M}$. Ryan and L. Shepley, Homogeneous Relativistic Cosmology (Princeton U.P., Princeton, NJ, 1975).

${ }^{13}$ I. M. Khalatnikov, E. M. Lifshitz, K. M. Khanin, L. N. Shchur, and Ya. G. Sinai, J. Stat. Phys. 38, 97 (1985); J. D. Barrow, Phys. Rep. C 85, 1 (1982); J. Pullin, in Proceedings of the SILARG VII Meeting, edited by M. Ryan, Jr. (to appear).

${ }^{14}$ S. W. Hawking, Phys. Rev. D 32, 2489 (1985); D. N. Page, Phys. Rev. D 32, 2496 (1985); H. D. Zeh, The Arrow of Time (Springer-Verlag, Hetdelberg, 1989).

${ }^{15} \mathrm{R}$. Penrose, in General Relativity, edited by S. W. Hawking and W. Israel (Cambridge U.P., Cambridge, 1979), p. 581. 